\title{
Vibration Analysis of 5-DOF Vehicle Model under Stochastic Road Surface Excitation
}

\author{
Yanlong Zhang ${ }^{1}$, Lixia Chen ${ }^{1}$, Li Wang ${ }^{2}$ and Wenxue Zhao ${ }^{1}$ \\ ${ }^{1}$ School of Mechatronic Engineering, Lanzhou Jiaotong University, Lanzhou 730070, China \\ ${ }^{2}$ Department of Mathematics, Lanzhou City University, Lanzhou 730070, China
}

\begin{abstract}
Considering human body vertical motion, vehicle body vertical motion, pitch movement and vertical jump of front and rear wheels, a five-degree-of-freedom vehicle model is established to study basic driving characteristics of the vehicle. Using Fourier transform method, acceleration power spectral density of the seat and the mean square value curves of seat vertical weighted acceleration are obtained by numerical simulation. Combined with comfort provision standards, the influence of vehicle model parameters and speed on seat acceleration power spectral density and vertical root-mean-square value of seat weighted acceleration are analyzed. Results show that the stiffness and damping of the seat have no significant effect on seat acceleration power spectral density, and seat acceleration PSD increases with increasing front or rear suspension stiffness, but it decreases with increasing front or rear suspension damping. It should also be concluded that the model stiffness and the mean square value of seat vertical weighted acceleration present positive correlation in general, but seat vertical weighted acceleration decrease first and then increase when model damping increase. Such analysis results can provide reference for the parameter optimization design of the automobile.
\end{abstract}

\section{Introduction}

Vehicle ride comfort is a major aspect of evaluating vehicle comfort properties. Commonly, 1/4 three degrees of freedom vehicle model does not take into account body pitching motion, vehicle sports performance does not reflect fully [1]. Therefore, it must to establish a multi-degree-of-freedom vehicle model, which can better reflect human, vehicle body and wheels vibration.

Many literatures have studied on a five-degree-offreedom (5-DOF) vehicle model. Xiaoliang Li analyzed 5 -DOF vibration of automobile ride comfort using pseudo-excitation method [2]. Lijun Zhang studied the non-stationary dynamics and control of 5-DOF vehicle [3]. Zhigang Huang analyzed 5-DOF mini-car vibration using calculation method [4]. Lixin Guo analyzed vehicle vibration in Changeable Speeds [5]. Jie Li found a kind of new method used for analyzing random vibration of vehicles [6]. But those studies did not analyze the effect of model parameter values on seat acceleration PSD and seat vertical root-mean-square value of weighted acceleration. Therefore, a 5-DOF vehicle vibration model which stochastic road surface excitation is conducted as the main source of vehicle vibration is established in this paper, and the ride comfort of the passengers is analyzed by frequency-domain simulation.

\section{The 5-DOF vehicle model}

As the vehicle suspension parts such as springs, shock absorbers and tires are symmetrical, a 5-DOF vehicle models used to describe automotive car vibration, which is shown in Figure 1, is established considering the manchair plane.

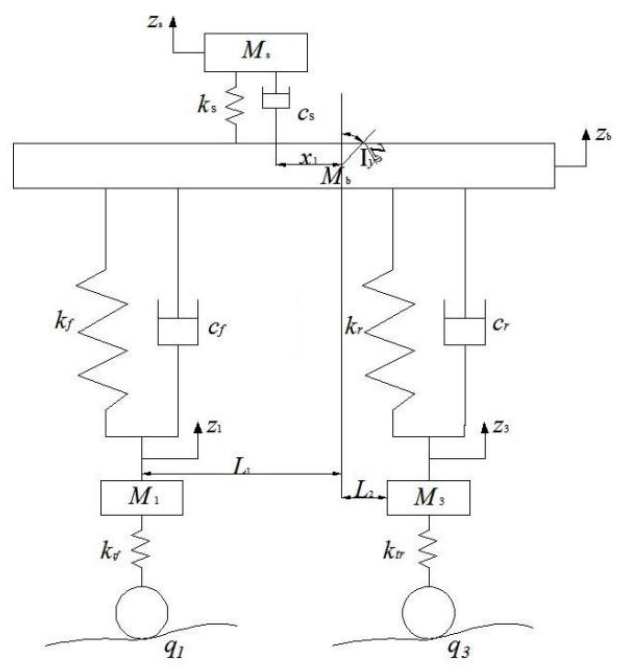

Figure 1. The 5-DOF vehicle model

In Figure $1, Z_{s}, Z_{b}, Z_{1}$ and $Z_{3}$ are the vertical displacement of seat, vehicle body, front, rear wheels. $Z_{p}$ is body pitch angle displacement. $q_{1}(t), q_{3}(t)$ are the front and rear wheels of road surface roughness. $M_{s}, M_{b}$,

Support by National Natural Science Foundation of China (11302092). 
$M_{1}, M_{3}$ are human-seat mass, vehicle body mass, front suspension mass, rear suspension mass, and their values are respectively $70 \mathrm{~kg}, 1100 \mathrm{~kg}, 50 \mathrm{~kg}, 65 \mathrm{~kg} . I_{y}, K_{s}$, $K_{f}, K_{r}, K_{t f}, K_{t r}$ are body moment inertia, seat stiffness, front suspension stiffness, rear suspension stiffness, front tyre stiffness and rear tyre stiffness, and their values are respectively $1840 \mathrm{~kg} . \mathrm{m}^{2}, 12000 \mathrm{~N} / \mathrm{m}, 17600 \mathrm{~N} / \mathrm{m}, 22300$ $\mathrm{N} / \mathrm{m}, 180000 \mathrm{~N} / \mathrm{m}$ and $180000 \mathrm{~N} / \mathrm{m} . C_{s}, C_{f}, C_{r}$ are damping coefficients of the seat, the front suspension, rear suspension, and their values are respectively 1250 $\mathrm{Ns} / \mathrm{m}, 1500 \mathrm{Ns} / \mathrm{m}, 1700 \mathrm{Ns} / \mathrm{m} . L_{1}, L_{2}, x_{1}$ are horizontal distance from body center mass to the front axle, the horizontal distance from body mass to the rear axle, horizontal distance from body center mass to the seat, and their values are respectively $1.30 \mathrm{~m}, 1.15 \mathrm{~m}, 0.779 \mathrm{~m}$.

According to the Lagrangian method, the differential equations of the system can be given by

$$
[M]\{\ddot{Z}\}+[C]\{\dot{Z}\}+[K]\{Z\}=[F] q
$$

where, $M=\operatorname{diag}\left[\begin{array}{lllll}M_{s} & M_{b} & I_{y} & M_{1} & M_{3}\end{array}\right], q(t)=\left[\begin{array}{l}q_{1}(t) \\ q_{3}(t)\end{array}\right]$,

$$
\begin{aligned}
C & =\left[\begin{array}{ccccc}
C_{s} & -C_{s} & C_{s} x_{1} & 0 & 0 \\
-C_{s} & C_{f}+C_{r}+C_{s} & -C_{s} x_{1}-C_{f} L_{1}+C_{r} L_{2} & -C_{f} & -C_{r} \\
C_{s} x_{1} & -C_{s} x_{1}-C_{f} L_{1}+C_{r} L_{2} & C_{s} x_{1}{ }^{2}+C_{f} L_{1}{ }^{2}+C_{r} L_{2}{ }^{2} & C_{f} L_{1} & -C_{r} L_{2} \\
0 & -C_{f} & C_{f} L_{1} & C_{f} & 0 \\
0 & -C_{r} & -C_{r} L_{2} & 0 & C_{r}
\end{array}\right], \\
K & =\left[\begin{array}{ccccc}
K_{s} & -K_{s} & K_{s} x_{1} & 0 & 0 \\
-K_{s} & K_{f}+K_{r}+K_{s} & -K_{s} x_{1}-K_{f} L_{1}+K_{r} L_{2} & -K_{f} & -K_{r} \\
K_{s} x_{1} & -K_{s} x_{1}-L_{1} K_{f}+L_{2} K_{r} & K_{s} x_{1}^{2}+L_{1}^{2} K_{f}+L_{2}{ }^{2} K_{r} & K_{f} L_{1} & -K_{r} L_{2} \\
0 & -K_{f} & K_{f} L_{1} & K_{f}+K_{t f} & 0 \\
0 & -K_{r} & -K_{r} L_{2} & 0 & K_{r}+K_{t r}
\end{array}\right], \\
Z & =\left[\begin{array}{llllll}
Z_{s} & Z_{b} & Z_{p} & Z_{1} & Z_{3}
\end{array}\right]^{T}, F=\left[\begin{array}{lllll}
0 & 0 & 0 & K_{t f} & 0 \\
0 & 0 & 0 & 0 & K_{t r}
\end{array}\right]^{T} .
\end{aligned}
$$

According to the literatures $[7,8]$, we can get $\dot{q}_{1}(t)+\beta V q_{1}(t)=\omega(t), \quad \dot{q}_{3}(t)=-\frac{2}{\Delta_{2}} q_{3}(t)+\left|\frac{2}{\Delta_{2}}+\beta V\right| q_{1}(t)-\omega(t)$, $\Delta_{2}=L / V$, where $q_{1}(t), q_{3}(t)$ is front and rear wheel road displacement; $\beta$ is road roughness coefficient; $V$ is vehicle speed; $\omega(t)$ is white noise; $\Delta_{2}$ is time delay; $L$ is wheelbase.

\section{Seat acceleration PSD}

Using Fourier transform method to derive system response power spectral density, we can get

$$
\begin{gathered}
\left(-\omega^{2}[M]+j \omega[C]+[K]\right)\{Z(\omega)\}=[F]\{q(\omega)\}, \\
{[H(\omega)]=\frac{\{Z(\omega)\}}{\{q(\omega)\}}=\left(-\omega^{2}[M]+j \omega[C]+[K]\right)^{-1}[F],}
\end{gathered}
$$

where, $\omega$ is circular frequency, $Z(\omega)$ is Fourier trasform of displacement vector $\{Z\}, q(\omega)$ is Fourier transform of road vectors $\{q\}, j=\sqrt{-1}$.
According to random theory, power spectral density of the vibration response can be obtained

$$
G_{x}(f)=|H(f)|_{x-q}^{2} G_{q}(f)
$$

where, $H(f)$ is amplitude-frequency characteristics of vibration responses when $\omega=2 \pi f, G_{q}(f)$ is power spectral density of the road. Seat vertical mean square value of weighted acceleration $a_{w k}$ can be calculated by

$$
a_{w k}=\left[\int_{0.5}^{80} W_{k}^{2}(f) G_{x}(f) d f\right]^{\frac{1}{2}}
$$

where, $W_{k}(f)=\left\{\begin{array}{ll}0.5 & (0.5<f<2) \\ f / 4 & (2<f<4) \\ 1 & (4<f<12.5) \\ 12.5 / f & (12.5<f<80)\end{array}\right.$.

The relationship between the total weighted acceleration RMS value and human subjective feelings is shown in Table $1[7,8]$.

Table 1. Relationship between $a_{w k}$ and human feelings.

\begin{tabular}{|c|c|}
\hline $\begin{array}{c}\text { Weighted acceleration } \\
\text { RMS } a_{w k} \mathbf{m} / \mathbf{s}^{\mathbf{2}}\end{array}$ & $\begin{array}{c}\text { Human subjective } \\
\text { feelings }\end{array}$ \\
\hline$<0.315$ & Comfortable \\
\hline $0.315 \sim 0.63$ & A little uncomfortable \\
\hline $0.5 \sim 1.0$ & Quite uncomfortable \\
\hline $0.8 \sim 1.6$ & uncomfortable \\
\hline $1.25 \sim 2.5$ & Very uncomfortable \\
\hline$>2.0$ & Extremely uncomfortable \\
\hline
\end{tabular}

We choose the $C$ level pavement, and the vehicle speed is $v=20 \mathrm{~m} / \mathrm{s}$. Using 4-order Runge-Kutta method, we can get the frequency domain response comparison graphs as shown in Figure 2. In Figure 2(a-b), the seat acceleration PSD has a tendency increase with the increase of front and rear suspension stiffness, the frequency of peak also increase, and the influence of front suspension stiffness take a larger proportion than back suspension stiffness. According to the calculation formula of seat natural frequency, we can get the natural frequency is $2.085 \mathrm{~Hz}$. In order to avoid the resonance of seats, front and back suspension, the stiffness can't be increased without any limit. But if the suspension stiffness of front and back is too small, it will lead to increase the suspension dynamic trip so that the passengers will feel uncomfortable. In Figure 2(c-d), the seat acceleration PSD will reduce with the increase of front and rear suspension damping. But the larger front and back suspension damping maybe leads the passengers to sustain more impact vibration, so suspension damping should be not too big. In Figure 2(e- 
f), the increasing of seat stiffness and damping have a little effect on the seat acceleration PSD, except for the resonance frequency area which should avoid.

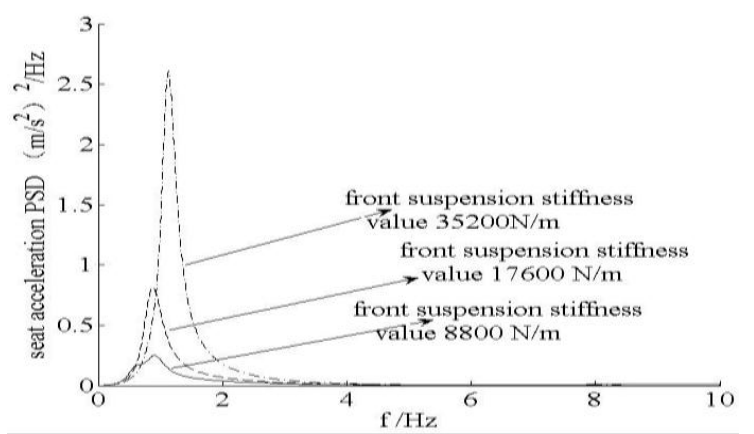

(a) The influence of the front suspension stiffness

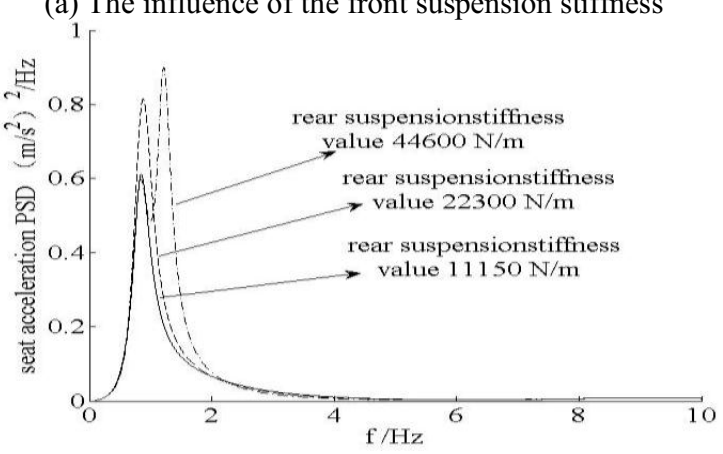

(b) The influence of the rear suspension stiffness

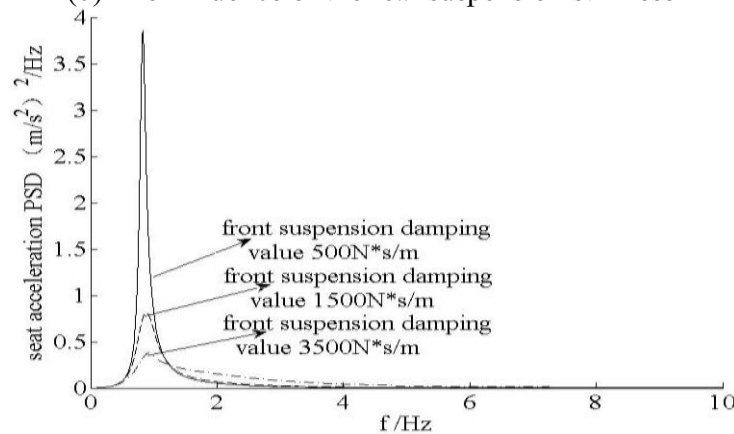

(c) The influence of the front suspension damping

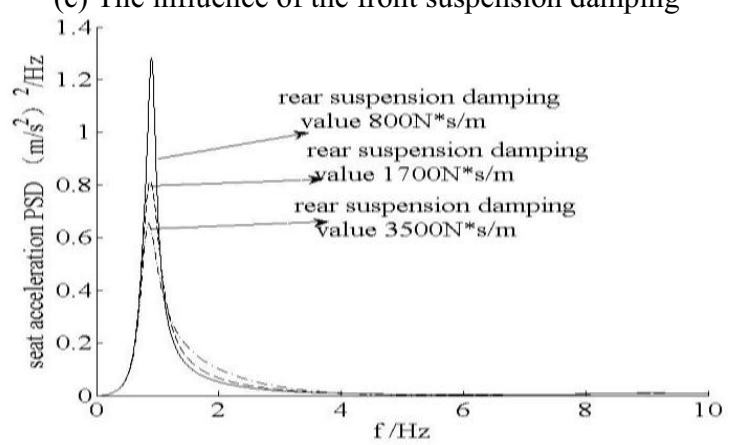

(d) The influence of the rear suspension damping

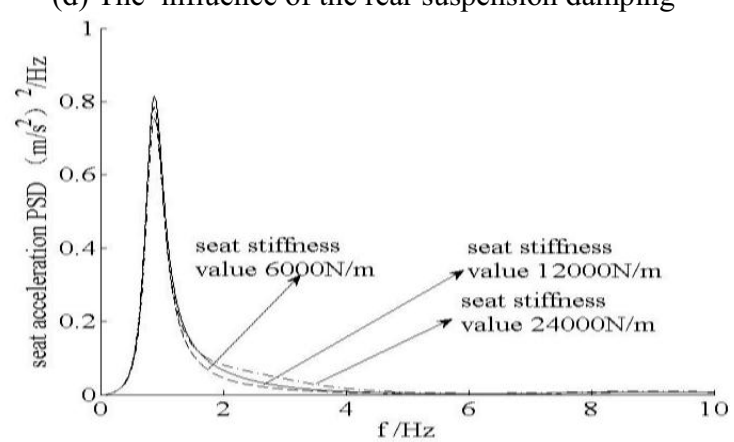

(e) The influence of the seat stiffnes

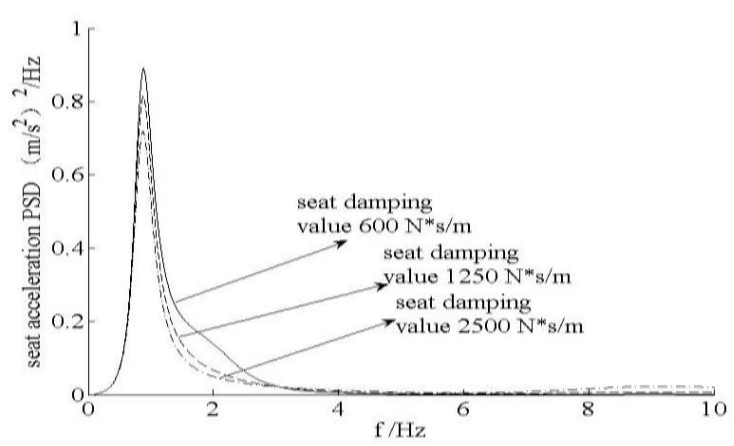

(f) The influence of the seat damping

Figure 2. Parameter's influence on seat acceleration PSD

\section{Seat weighted acceleration RMS}

The influence of vehicle parameters and speed on the root mean square value of weighted acceleration of the seat is analyzed in Figure 3.

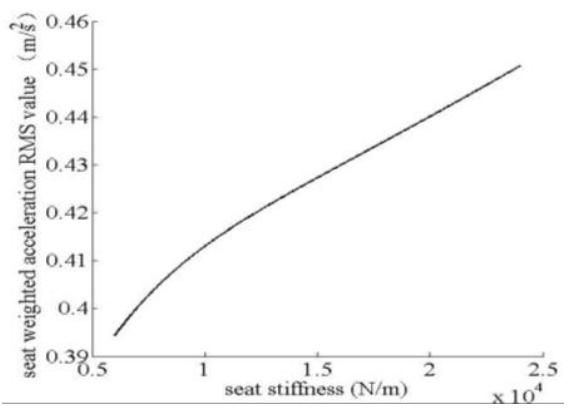

(a) The influence of the seat stiffness

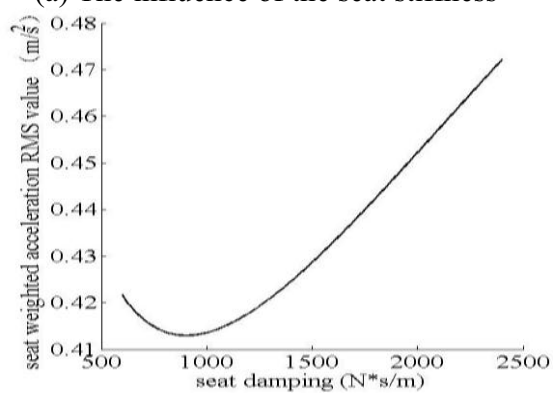

(b) The influence of the seat damping

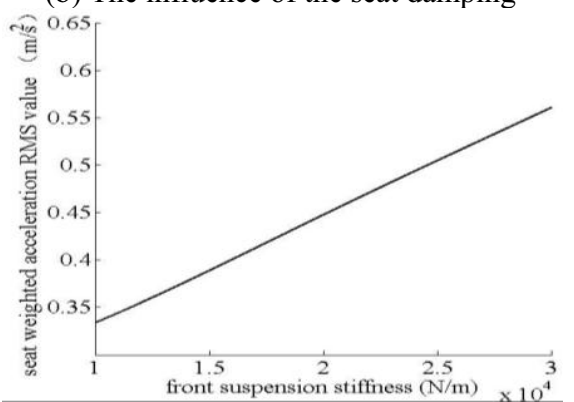

(c) The influence of the front suspension stiffness

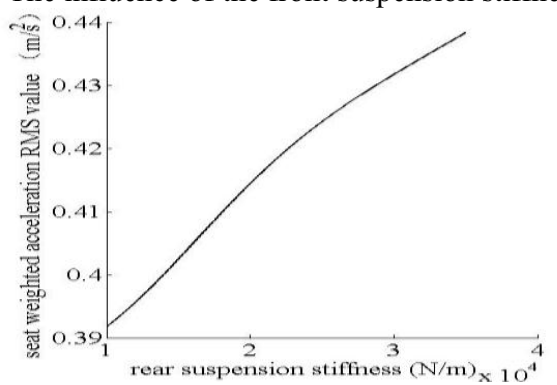

(d) The influence of the rear suspension stiffness 


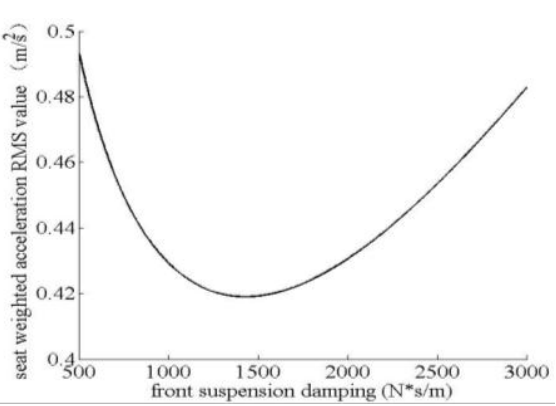

(e) The influence of the front suspension damping

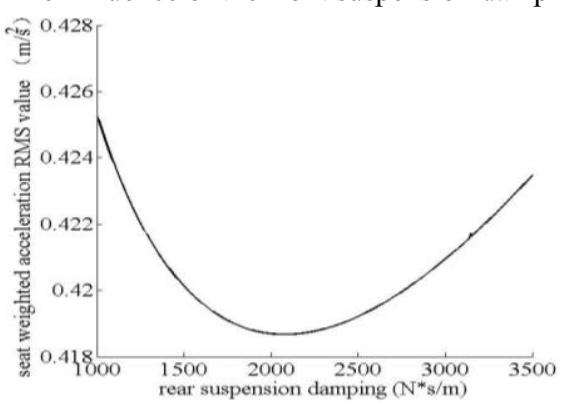

(f) The influence of the rear suspension damping

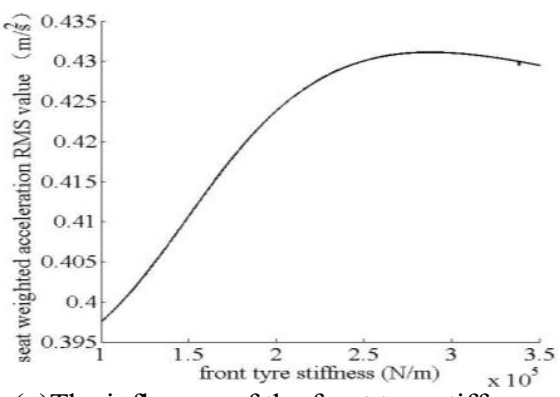

(g)The influence of the front tyre stiffness

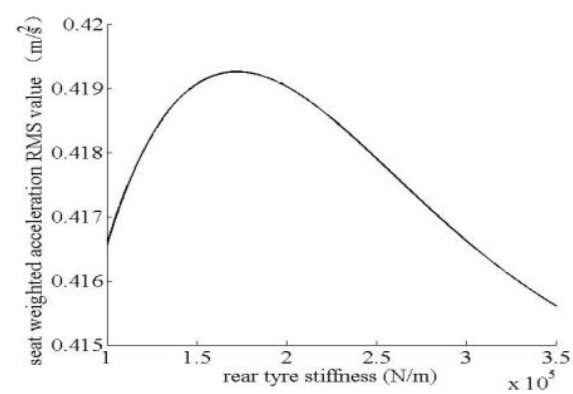

(h)The influence of the rear tyre stiffness

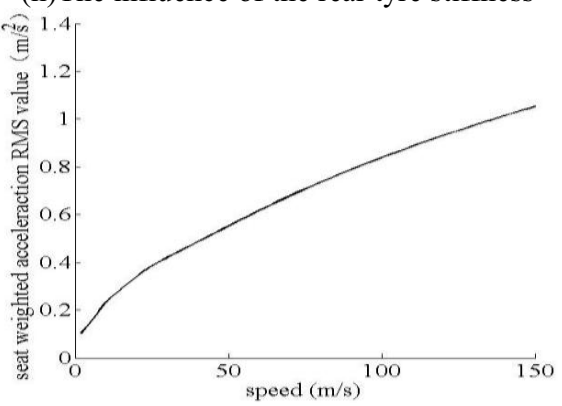

(i) The influence of the vehicle speed

Figure 3. Parameter's influence on the seat vertical weighted acceleration RMS

With increasing the stiffness of seat, front suspension, rear suspension and the vehicle speed, the root-meansquare value of weighted acceleration $a_{w k}$ increases as shown in Figure 3(a), (c), (d) and (i). With increasing the damping of seat, front and rear suspension, $a_{w k}$ decreases first and then increases as shown in Figure 3(b), (e) and (f). With increasing the stiffness of front and rear tyre, $a_{w k}$ increases first and then decreases as shown in Figure $3(\mathrm{~g})$ and $(\mathrm{h})$. Referred to table 1 , the suitable vehicle parameter ranges and the suitable vehicle speed range can be obtained according to the comfort requirements of the passengers. The above analysis results can provide the reference for the vehicle's parameter optimization design.

\section{Conclusions}

Considered the stochastic road surface excitation, a 5DOF vehicle model is established to study basic driving characteristics of the vehicle. The acceleration power spectral density of the seat and the mean square value curves of seat vertical weighted acceleration are obtained at different system parameters by numerical simulation. Combined with the comfort provision standards ISO2631-1:1997(E), the influence of vehicle model parameters and speed on seat acceleration power spectral density and vertical root-mean-square value of seat weighted acceleration are analyzed. Results show that the stiffness and damping of the seat have no significant effect on seat acceleration power spectral density, and seat acceleration PSD increases with increasing front or rear suspension stiffness, but it decreases with increasing front or rear suspension damping. It should also be concluded that the model stiffness and the mean square value of seat vertical weighted acceleration present positive correlation in general, but seat vertical weighted acceleration decrease first and then increase when model damping increase. The suitable vehicle parameter ranges and the suitable vehicle speed range can be obtained according to the comfort requirements of the passengers. Such analysis results can provide the reference for the vehicle's parameter optimization design.

\section{References}

1. Hongliang Zhang, Binggang Wang, Wanqiao Yang, J. of Chongqing Jiaotong Univ. (Natural Science), 28:6, 1041 (2009).

2. Xiaoliang Li, Vibration simulation and analysis for automobile ride comfort based on pseudo excitation method(Wuhan univ. of technology, 2011).

3. Lijun Zhang, Study on non-stationary ride dynamics and control of vehicle(Northeastern univ., 2006).

4. Zhigang Huang, Muqiao Zheng, J. of Beijing Institute of Light Industry, 13:1, 68 (1995).

5. Lixin Guo, Liping Zhang: Mathematical Problems in Engineering, 2010, 1 (2010).

6. Jie Li, Yuyin Qin, Qi Zhao, Wei Zhang, J. of Machine Design, 26:4, 14(2009)

7. P Michelberger, L Palkovics, J Bokor, Int. J. of Vehicle Design, 14:22, 145(1993).

8. F Oueslati, S Sankar, SAE, 534(1994). 